Check for updates

Cite this: RSC Adv., 2017, 7, 55790

Received 2nd November 2017

Accepted 1st December 2017

DOI: $10.1039 / c 7 r a 12034 f$

rsc.li/rsc-advances

\title{
Influence from the types of surface functional groups of RGO on the performances of thermal interface materials $\uparrow$
}

\author{
Yunfei Sun, (D) *a Yanfeng He, ${ }^{\mathrm{b}}$ Bo Tang, (D) *b Chongben Tao, ${ }^{\mathrm{a}}$ Jianmin Ban ${ }^{\mathrm{a}}$ \\ and Li Jiang ${ }^{a}$
}

\begin{abstract}
RGO nanosheets-modified epoxy resin (ER) is a major research interest because of the high thermal performance of the resulting thermal interface materials (TIMS). In this study, the attention is focused on the influences from the types of functional groups of the RGO nanosheets on the obtained thermal conductivity. Among all the types of functional groups, the carboxyl group is found to play the most important role to enhance the phonon transport at the interface between the graphene basal plane and ER, which is proven by the obtained thermal conductivity and calculated thermal boundary resistance. A close chemical contact between the graphene and ER is one of the key factors in the resulting high thermal conductivity. Moreover, the formed chemical bond based on the carboxyl group from the RGO and hydroxyl (epoxy) group from the ER also exerts a positive influence on the resulting mechanical performance, which endows a satisfactory ultimate strength and stretch limits to the as-prepared TIMs. The findings of this study reveal the core factors which determine the obtained performances of the graphene-assisted ER, providing a path to further enhance the thermal and mechanical properties of the TIMs for practical application.
\end{abstract}

\section{Introduction}

Graphene-modified thermal interface materials (TIMs) have attracted increasing attention because their high thermal and mechanical performances can improve the corresponding interface performances of electronic components as a result of the unique electric and mechanical properties of graphene. ${ }^{\mathbf{1 - 4}}$ Based on reduced graphene oxide (RGO) and three-dimensional graphene networks (3DGNs, prepared by the chemical vapor deposition method), various studies of graphene-assisted epoxy resin (ER, routinely used as the matrix of TIMs because of its non-toxicity, corrosion resistance and cheapness) have been carried out. ${ }^{5-7}$ Sung et al. used RGO in combination with ER, and the thermal conductivity increased from $0.2 \mathrm{~W} \mathrm{~m}^{-1} \mathrm{~K}^{-1}$ for the pristine ER to $1.5 \mathrm{~W} \mathrm{~m}^{-1} \mathrm{~K}^{-1}$ for the prepared composites. ${ }^{5}$ Balandin's group further optimized the employed RGO to improve the thermal and mechanical performances of the RGOER composite (the thermal conductivity reached $\sim 4 \mathrm{~W} \mathrm{~m}^{-1}$ $\left.\mathrm{K}^{-1}\right){ }^{6}$ Recently, our group found that 3DGNs may be a better choice to be adopted as the thermal filler due to the lower defect

${ }^{a}$ College of Electronic and Information Engineering, Suzhou University of Sciences and Technology, Suzhou, Jiangsu 215009, People's Republic of China

${ }^{b}$ School of Petroleum Engineering, Changzhou University, Changzhou city 213016, China.E-mail: 113965203@qq.com; tangbo@cczu.edu.cn

$\dagger$ Electronic supplementary information (ESI) available. See DOI: 10.1039/c7ra12034f density and larger average size, and the resulting thermal conductivity of the 3DGNs-ER is about $20 \%$ higher than in the case of adopting an RGO filler with an identical mass fraction. ${ }^{7}$ However, the thermal boundary resistance $(\delta)$ between 3DGNs and ER is much higher than that between RGO and ER, and the core reason is that the residual surface functional groups on the RGO surface play a positive role (acting as a bridge) to improve the phonon transport at the interface between the graphene basal plane and ER. ${ }^{7,8}$

In order to utilize the advantages of 3DGNs (which possess a lower Umklapp resistance), a further chemical tailoring of this material is needed to enhance the wettability between the filler and matrix. ${ }^{9}$ However, adjusting the defect density of the 3DGNs (the presence of defects endows a better phonon transport ability at the interface area) is rather complex (including an extremely high gas flow and a strictly controlled cooling rate). ${ }^{\mathbf{1 0}}$ In contrast to the tedious processing needed to control the defect density of 3DGNs, the adjustment of the amount of functional groups on the RGO surface can be achieved easily by changing the reduction time. ${ }^{\mathbf{1 1 - 1 3}}$ Therefore, optimizing the RGO structure is considered a more feasible way to prepare TIMs on a large scale. Recently, our group proposed a comprehensive criterion to judge the performance of the adopted RGO in TIMs, including its mass fraction, reduction degree, average size and thickness. ${ }^{14}$ Therein, the reduction degree of the RGO is the determinant of the resulting thermal performance of the TIMs. As is known, three types of functional groups including 
hydroxyl, carboxyl and epoxy groups appear on the graphene oxide (GO) surface, and part of these groups remain after the reduction procedure and act as a bridge between the filler and matrix. ${ }^{14,15}$ However, which type of functional group makes the major contribution to enhance the phonon transport at the interface is still unclear, deserving further study.

In this study, the type and residual amount of functional groups on the RGO surface are controlled by selecting various oxidation and reduction agents, as well as adjusting the reduction time. Then, these thermal fillers are adopted to modify ER, in order to reveal the influence from each type of functional group on the resulting thermal performance of the TIMs. The Kapitza resistance (Kapitza boundary scattering) can be remarkably reduced by optimizing the reduction degree and functional group type of the adopted RGO in order to form a strong chemical contact between the graphene basal plane and ER. The study provides a path to further improve the thermal performance of the graphene-assisted TIMs. Moreover, the mechanical properties of the resulting TIMs are also tested, and are found to satisfy the requirements for practical application.

\section{Results and discussion}

SEM images of the pristine ER and resulting TIMs are shown in Fig. 1, and the mass fractions of these fillers are identical (5 wt\%). These composites are labeled as RGO (h, e)-ER, RGO (x, e)-ER, RGO (h, h)-ER and RGO (x, h)-ER according to the adopted oxidation and reduction agent, respectively. Some obvious micropores can be seen in the surface and interior of the original ER (Fig. 1a), which increase the thermal resistance of the material. In contrast, the similar smooth surface and interior of the prepared composites indicate the removal of pores during the mixing and solidification processes after adding the RGO samples (Fig. 1b-e), implying that all of the composites should possess relatively high thermal conductivities. The EDS results of random positions from these resulting TIMs are shown in Fig. 1f. Hydrazine removed all kinds of functional groups on the RGO surface, while only epoxy groups were eliminated when ethylene was used as the reduction agent. ${ }^{7,14,17}$ Therefore, the contents of carbon in RGO (h, h)-ER and RGO (x, h)-ER are much higher than that of the RGO (h, e)ER and RGO (x, e)-ER.

As a non-destructive characterization technique, Raman spectroscopy is widely used to identify the thickness, average size and defect density of graphene samples. Three fingerprint signals, the G, D and 2D peaks, can be found in the corresponding curves from the graphite-like materials. ${ }^{20-22}$ The $\mathrm{G}$ band located at $\sim 1600 \mathrm{~cm}^{-1}$ results from $E_{2 g}$ phonons at the Brillouin zone centre, while the $\mathrm{D}$ band is associated with the concentration of defects (the peak disappears when a pristine sample is employed). ${ }^{22}$ Interestingly, the $2 \mathrm{D}$ band is independent of defects but sensitive to the number of graphene layers, although it is the second order of the D band. Raman curves of the various RGO specimens are displayed in Fig. 2, and the relative intensities of the $\mathrm{D}$ peak from the RGO (h, e) and RGO $(\mathrm{x}, \mathrm{e})$ are stronger than that of the RGO $(\mathrm{h}, \mathrm{h})$ and RGO $(\mathrm{x}, \mathrm{h})$,
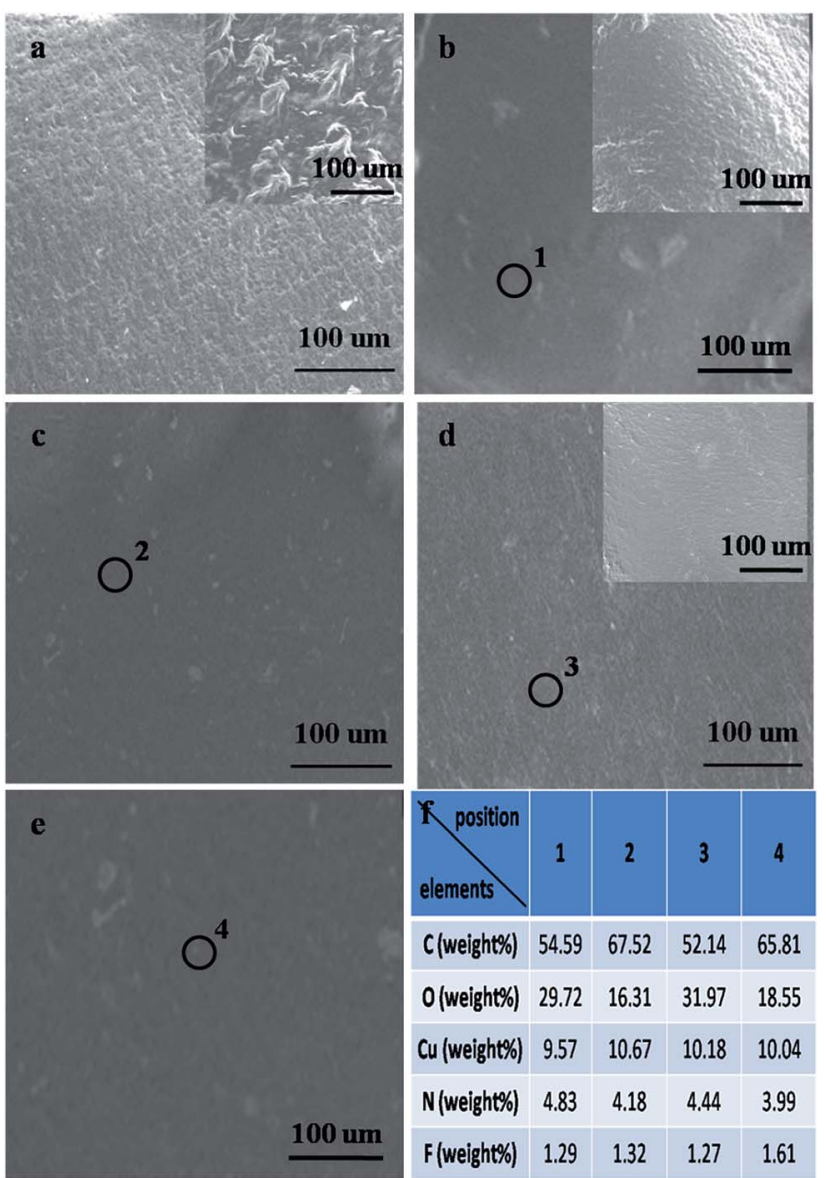

Fig. 1 (a-e) SEM images of pure ER, RGO (h, e)-ER, RGO (h, h)-ER, $R G O(x, e)-E R$ and RGO $(x, h)-E R$. The mass fractions of fillers in these samples are 5 wt\%. Insets show the cross-section view of the samples; (f) EDS results of random positions of these samples (indicated by circles)

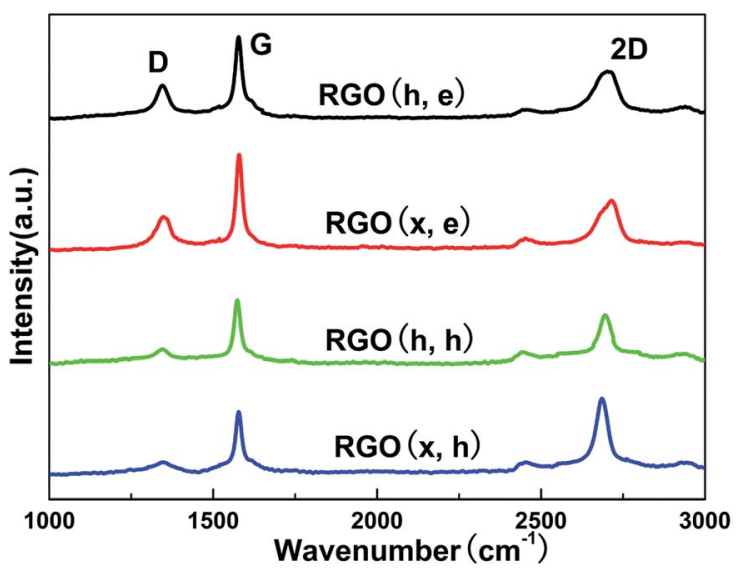

Fig. 2 Raman curves of various RGO fillers.

indicating the higher functional group density of the former, which is in line with the EDS results.

XPS is a useful tool to obtain information on the chemical states of carbon atoms in GO and RGO samples, and the 
proportions of carbon atoms from elemental carbon and various functional groups can be calculated. The corresponding curves of the GO (h) and GO (x) are displayed in Fig. $3 \mathrm{a}$ and $\mathrm{b}$. Two major peaks located at $284.7 \mathrm{eV}$ and $288.8 \mathrm{eV}$, which result from elemental carbon and carbon atoms from $\mathrm{HO}-\mathrm{C}=\mathrm{O}$, can be seen. ${ }^{7,21,23}$ The contribution of carbon atoms from $\mathrm{C}=\mathrm{O}$ and $\mathrm{C}-\mathrm{OH}$ can also be found at $287.4 \mathrm{eV}$ and $285.9 \mathrm{eV}$ after an elaborate fitting. ${ }^{23}$ A significant difference in the relative intensities of carbon atoms in various chemical states can be observed between these two profiles. Therein, the ratio is 42 : 14 : 7 : 37 (elemental carbon, carbon atoms from hydroxyl, epoxy and carboxyl groups) for the GO (h). For the GO (x), the proportion of carbon atoms from $\mathrm{C}-\mathrm{OH}$ increases to $\sim 39 \%$ (almost three times higher than that of the GO (h)). The observed differences between the XPS curves result from the various oxidation processes (more details in the Experimental section). For the GO (h), the traditional Hummers' method introduces more carboxyl groups. For the GO (x), hydroxyl rather than carboxyl becomes the major functional group by using the approach reported by Zhang et al. ${ }^{17}$ Two values, including $m_{\mathrm{C}} /$ $m_{\mathrm{O}}$ and $C_{\text {element }} / C_{\text {functional }}$, were used to estimate the oxidation (or reduction) degree of the GO (or RGO) samples. According to the values of $C_{\text {element }} / C_{\text {functional }}(1 / 1.08$ for both the GO (h) and GO (x)) and $m_{\mathrm{C}} / m_{\mathrm{O}}(0.789$ for the GO (h) and 1.087 for the GO $(\mathrm{x})$ ), the total functional group amounts of the GO (x) and GO (h) are identical. In other words, these two samples possess a similar oxidation degree (the total amounts of the various functional groups are identical). Moreover, the total amounts of acidic groups of the GO and RGO samples were determined by a titration method reported by Sheibani and Heydari. ${ }^{24-26}$ On the basis of these tests, the total amounts of acidic groups of all the prepared samples are listed in Table 1 , and the GO (h) and RGO (h, e) possess the highest values. In order to confirm the results, the adsorption capacities of these specimens for cationic dye (methylene blue was used as the adsorbate) were also detected. ${ }^{24}$ The corresponding results are shown in Table 1 , and the GO (h) and RGO (h, e) display the highest adsorptivity, further proving that these specimens possess the most acidic groups. The thermal performances of the GO (x)-ER and GO (h)-ER are shown in Fig. 4a, and all of the products display improved thermal conductivities compared to that of the original ER (and the obtained values from the GO (h)-based samples are higher than that of the GO (x)-added samples as a whole). However, the high defect density of the fillers limits the further enhancement of the resulting thermal conductivities. Based on Balandin's
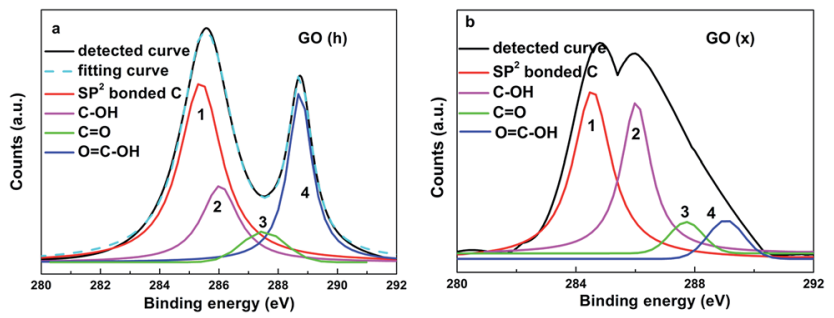

Fig. 3 XPS curves of (a) GO (h) and (b) GO (x).
Table 1 Total amounts of acidic groups and adsorption abilities of various samples

\begin{tabular}{|c|c|c|}
\hline \multirow[b]{2}{*}{ Samples } & \multicolumn{2}{|l|}{ Properties } \\
\hline & $\begin{array}{l}\text { Total amount of } \\
\text { acidic groups }\left(\mathrm{mmol} \mathrm{g}^{-1}\right)\end{array}$ & $\begin{array}{l}\text { Residual amount } \\
\text { of the MB (\%) }\end{array}$ \\
\hline GO (h) & 25.5 & 91.4 \\
\hline GO (x) & 22.0 & 92.9 \\
\hline RGO (h, e) & 13.6 & 95.1 \\
\hline RGO (h, h) & 10.8 & 96.2 \\
\hline RGO (x, e) & 13.1 & 95.4 \\
\hline RGO (x, h) & 11.2 & 96.4 \\
\hline
\end{tabular}
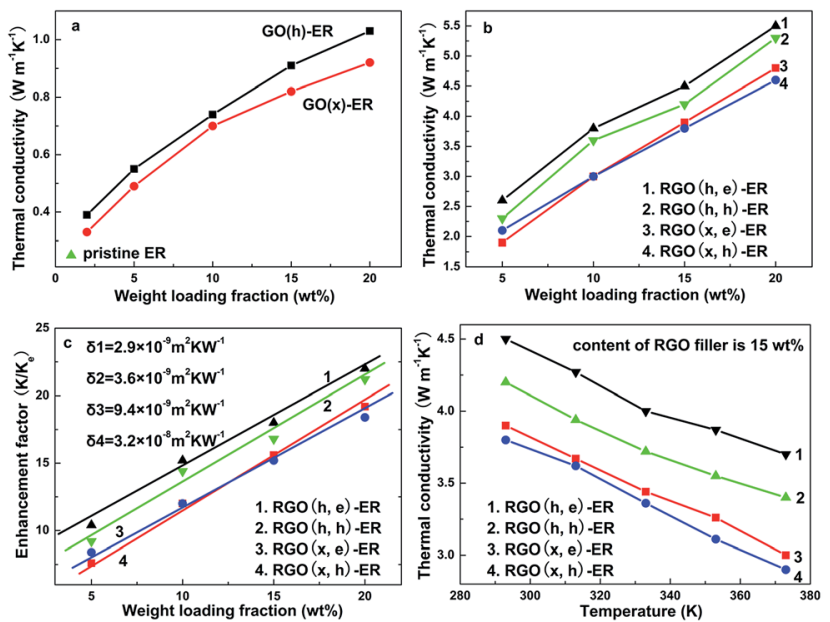

Fig. 4 Thermal conductivities of (a) GO and (b) RGO-modified ER with various mass fractions; (c) thermal boundary resistances of various RGO-added ER and (d) thermal stability of resulting TIMs under high temperatures.

and Duong's studies, the thermal conductivity of graphenebased composites can be calculated by the following equation: ${ }^{27-30}$

$$
K=K_{\mathrm{g}}\left[\frac{2 p\left(K_{\mathrm{g}}-K_{\mathrm{e}}\right)+3 K_{\mathrm{e}}}{(3-p) K_{\mathrm{g}}+K_{\mathrm{e}} p+\frac{\delta K_{\mathrm{g}} K_{\mathrm{e}} p}{H}}\right]
$$

where $p$ represents the volume percentage of the graphene filler, $K, K_{\mathrm{g}}$ and $K_{\mathrm{e}}$ are the thermal conductivities of the resulting composite, graphene and ER, respectively. $H$ and $\delta$ are the thickness of the adopted RGO sample and thermal boundary resistance between the matrix and filler, respectively. Considering the relatively small size and thickness of the adopted RGO, the practical $K_{\mathrm{g}}$ should be much smaller than the theoretical value $\left(5000 \mathrm{~W} \mathrm{~m}^{-1} \mathrm{~K}^{-1}\right)$. Based on Fan et al., $100 \mathrm{~W} \mathrm{~m}^{-1}$ $\mathrm{K}^{-1}$ is a reasonable value. ${ }^{28}$

The calculated $\delta$ of the GO (h)-modified samples are lower than that of the GO $(\mathrm{x})$-based products $\left(4.1 \times 10^{-9} \mathrm{~m}^{2} \mathrm{KW}^{-1}\right.$ for the GO (h)-ER sample and $9.4 \times 10^{-9} \mathrm{~m}^{2} \mathrm{~K} \mathrm{~W}^{-1}$ for the GO (x)ER sample), which brings about the observed difference in the thermal performances. These calculated values are comparable with the previous report, indicating that the adopted model is 
reasonable. ${ }^{28}$ Therefore, the types of functional groups on the graphene surface exert a remarkable influence on the interface thermal contact (the oxidation degrees of the GO (x) and GO (h)based samples are almost identical). Therein, the carboxyl and epoxy groups rather than hydroxyl group make the major contribution as a bridge for the phonon transport at the interface area (according to the XPS curves of the GO (x) and GO (h)). In order to further confirm this point, the RGO (h, e), RGO (x, e), RGO (h, h) and RGO (x, h) were used to modify the ER. Considering that an identical reduction degree is one of the preconditions for a meaningful comparison, the total amounts of functional groups of these samples were adjusted to the same value (the corresponding calculated results of $m_{\mathrm{C}} / m_{\mathrm{O}}, C_{\text {element }} /$ $C_{\text {functional }}$ and the ratios of various functional groups of all the samples, based on XPS curves, are shown in Table 2). Therefore, the major distinction among these adopted fillers is the designed ratios of various functional groups. ${ }^{18}$ When using ethanol as the reduction agent, only the epoxy groups are removed while carboxyl and hydroxyl groups are retained. In contrast, all three types of functional groups are eliminated simultaneously when hydrazine is adopted. Based on the above discussion, the types and corresponding ratios of the surface functional groups can be adjusted by selecting the oxidizing and reducing agents. Briefly, the oxidants determine the introduced functional groups (for the GO), while the reductants govern the residual functional groups (for the RGO). Comparing the obtained thermal conductivities and calculated $\delta$ of these prepared TIMs (Fig. 4b and c), it can be found that the $\delta$ of the RGO (x, e)-ER and RGO (x)-ER, as well as RGO (h, e)-ER and RGO (h)-ER are similar, indicating that the reduction of epoxy groups does not markedly affect the phonon transport at the interface. On the other hand, the $\delta$ increases significantly when the adopted GO samples are reduced by hydrazine. Therefore, the most important functional group of the RGO to improve the interface properties is the carboxyl group.

The fundamental reason for the better contact at the interface area is a closer chemical interaction between the filler and matrix. Therefore, the contact between the various fillers and ER should be different, which can be ascertained from the IR spectra. For the pristine ER, several obvious fingerprint absorption peaks, i.e. rocking of $-\mathrm{CH}_{2}$, stretching of $\mathrm{C}-\mathrm{O}-\mathrm{C}$, deformation of $\mathrm{C}-\mathrm{H}$, and stretching of $\mathrm{C}-\mathrm{H}$ and $\mathrm{C}-\mathrm{OH}$, located at 772, 831, 1390, 3000 and $3380 \mathrm{~cm}^{-1}$, can be seen (Fig. 5). ${ }^{31-33}$ The major absorption peaks of the RGO samples corresponding to $\mathrm{C}-\mathrm{OH}, \mathrm{O}=\mathrm{C}-\mathrm{OH}$ and $\mathrm{C}-\mathrm{C}$ (skeletal vibration of the graphene basal plane) are observed at 1179, 1380 and $1600 \mathrm{~cm}^{-1}$,

Table 2 Ratios of carbon atoms in various chemical states in all the RGO samples

\begin{tabular}{llll}
\hline Samples & $C_{\text {element }}: C_{\text {functional }}$ & $m_{\mathrm{C}}: m_{\mathrm{O}}$ & $\begin{array}{l}C_{\text {element }}: C_{-\mathrm{OH}}: \\
C_{=\mathrm{O}}: C_{-\mathrm{OOH}}\end{array}$ \\
\hline RGO (h, e) & $1.94: 1$ & $1.19: 1$ & $66: 5: 0: 29$ \\
RGO (h, h) & $11.5: 1$ & $5.77: 1$ & $92: 2: 1: 5$ \\
RGO (x, e) & $1.63: 1$ & $1.59: 1$ & $62: 28: 1: 9$ \\
RGO (x, h) & $15.7: 1$ & $9.38: 1$ & $94: 4: 0: 2$
\end{tabular}

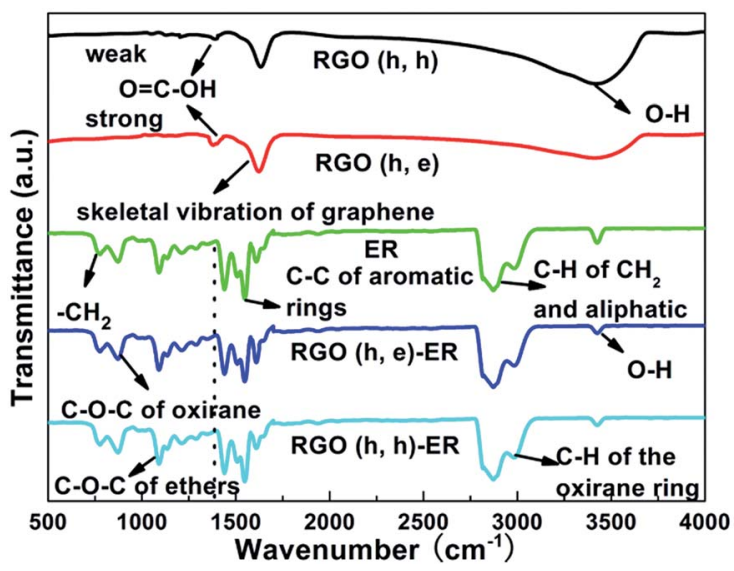

Fig. 5 IR curves of various RGO samples, pristine ER and resulting TIMs.

respectively. ${ }^{34}$ The integrated intensity of carboxyl groups from the RGO (h, e) is much stronger than that of the RGO $(h, h)$, which is in line with the XPS results. Moreover, the broad absorption peak ranging from 3000 to $3700 \mathrm{~cm}^{-1}$ is attributed to the $\mathrm{O}-\mathrm{H}$ stretching vibration of adsorbed water. ${ }^{35}$ After combining with the ER, the absorption peak belonging to $\mathrm{O}=$ $\mathrm{C}-\mathrm{OH}$ disappears (shown by a dotted line), indicating that the formation of chemical bonds between these RGOs and ER during the mixing and solidification processes is owing to the carboxyl group. ${ }^{36}$ A plausible explanation of the above obtained result (the disappearance of the $\mathrm{O}=\mathrm{C}-\mathrm{OH}$ peak) is that the major functional groups of the ER are epoxy and hydroxyl groups, which react with the carboxyl of the RGO fillers (the signal intensity of the $\mathrm{C}-\mathrm{OH}$ absorption band from the ER decreases in the composites). Therefore, the greater number of carboxyl groups endows the RGO (h, e) (derived from GO (h)) sample a greater ability to form strong chemical bonds with the ER than the equivalent ability of the RGO (x, e) (derived from GO $(\mathrm{x})$ ), which leads to a smaller $\delta$ at the interface field.

Although a small $\delta$ manifests in a better contact between the graphene basal plane and ER, the obtained thermal conductivity is not only determined by this parameter. There are three sources for the thermal resistance of the resulting TIMs, including resistance from the ER, graphene basal plane and their interface area. Adopting an identical mass fraction of the fillers, we have proven that the thermal resistances from the ER are identical. ${ }^{\mathbf{1 4}}$ Therefore, the difference in the resulting thermal resistance results from the graphene basal plane and interface area (Umklapp resistance and Kapitza resistance). ${ }^{7}$ Due to their origins, the former (Umklapp resistance) increases with elevated temperature, while the latter decreases. ${ }^{37,38}$ Methods for the further optimization of the adopted RGO include controlling the thickness, adjusting the average size and decreasing the defect density (far away from the boundary of the graphene basal plane). Greater size and lower defect density decrease the Umklapp resistance and increase the mean free path of phonons, which improves the thermal performance of the resulting TIMs. Moreover, the reduction degree of the adopted RGO is closely related to the obtained thermal 
conductivity. Although a large amount of carboxyl groups can provide a bridge to achieve better phonon transport ability at the interface between the graphene basal plane and ER, the good intrinsic thermal property is degraded. Therefore, optimizing the type of functional group is quite important for the thermal performance of the resulting TIMs when the reduction degree of the RGO is limited.

Thermal conductivity stability under high temperature is one of the important properties of TIMs for their practical application. The resulting thermal performances under increasing temperatures of these as-prepared samples are shown in Fig. 4d. Under high temperature, the performances of all the TIMs degrade remarkably, and about a 20\% decrease in the thermal conductivity for each sample can be found at $100{ }^{\circ} \mathrm{C}$ compared to that under room temperature. However, the resulting thermal conductivity is still satisfactory for practical application. Moreover, all the TIM samples show similar thermal stability under high temperature, indicating that the stability is not dependent on the type of functional groups of the adopted RGO specimens. In fact, the composite should display a better thermal stability when the employed RGO sample possesses a smaller average size (because the increased Umklapp resistance can be partly compensated by the decreased Kapitza resistance under high temperature resulting from the extra boundary fields), which has been confirmed by our previous reports. ${ }^{7,14}$ However, the extra boundary areas will have a negative effect on the thermal conductivity as a result of the degraded intrinsic thermal performance. Therefore, morphology is another important factor to obtain an optimized thermal performance, ${ }^{\mathbf{1 4}}$ and a three-layer structure, $117 \mathrm{~nm}$ average size and low defect density of the RGO are recommended by our previous reports. After extensive experiments, the optimum total amount of functional groups on the RGO was found to be $m_{\mathrm{C}}: m_{\mathrm{O}}=7.4: 1, C_{\text {element }} / C_{\text {functional }}=14.8: 1(\mathrm{a} 4 \mathrm{~h}$ reduction process of the $\mathrm{RGO}(\mathrm{h}, \mathrm{e})$ is recommended). Therein, the percentage of carboxyl groups is higher than 60\% (among the three types of functional groups), leading to a much higher thermal conductivity than that of other samples (with $20 \mathrm{wt} \%$ RGO filler, the thermal conductivity is $5.9 \mathrm{~W} \mathrm{~m}^{-1} \mathrm{~K}^{-1}, 29.5$ times higher than the pristine ER and $11.3 \%$ higher than our previous reported TIMs prepared without optimizing the type of surface functional groups). ${ }^{14}$ This again confirms that the carboxyl group is the key factor for the phonon transport at the interface area.

Moreover, mechanical performance is also a vital characteristic for the practical application of the TIMs. The influence of the added RGO sample on the ultimate strengths and stretching limits was investigated, and the stress-strain curves and corresponding data are displayed in Fig. S1 and Table S1 in the ESI. $\uparrow$ Based on the good repeatability of the test results, the decrease of the ultimate strength after adding the RGO fillers can be neglected. Similarly, the stretching limits of the resulting TIMs are comparable to that of the pristine ER. Moreover, it was found that a better mechanical performance was obtained when the RGO specimens possessed a higher ratio of carboxyl groups (with the total amount of functional groups constant), indicating that a stronger connection between the graphene basal plane and ER can be achieved through carboxyl groups compared with other functional groups. Therefore, a high ratio of carboxyl groups is an optimum choice for both the thermal and mechanical performances of the TIMs.

\section{Experimental}

\section{Materials}

Ethanol, hydrochloric acid, potassium permanganate, hydrazine peroxide, methylene blue $(\mathrm{MB})$ and sulfuric acid were purchased from the Beijing chemical reagent plant (Beijing, China). Natural graphite and acetone were received from Aladdin Co., Ltd. ER and curing agent were obtained commercially from Sanmu Co. Ltd. (Suzhou, China). Deionized water (resistivity $18 \mathrm{M} \Omega \mathrm{cm}$ ) was utilized to prepare all aqueous solutions.

\section{Preparation}

The graphene oxide (GO) samples were prepared by the modified Hummers' method and the approach reported by Zhang, and the obtained GO products are labeled as GO (h) and GO (x), respectively. ${ }^{16,17}$ Two methods were used to reduce the GO samples, obtaining RGO samples with various reduction degrees. For the first method, reported by $\mathrm{Xu}$ et al., alcohol is used as the reduction agent to react with the GO (h) and GO (x). ${ }^{18}$ Briefly, a certain amount of GO sample was dispersed in $50 \mathrm{~mL}$ of ethylene glycol and a $60 \mathrm{~min}$ sonication treatment was performed. Then, the suspension was heated to $160{ }^{\circ} \mathrm{C}$ for $5 \mathrm{~h}$ under vigorous stirring. After a subsequent centrifugation process, the sample was washed by deionized water 3 times. Lastly, the obtained paste was dried at $60{ }^{\circ} \mathrm{C}$ in a vacuum oven (the obtained products named RGO (h, e) and RGO (x, e)). In the second method, hydrazine is employed to reduce the GO samples. Briefly, $2 \mathrm{~mL}$ hydrazine was added into a $30 \mathrm{~mL} \mathrm{GO}$ solution $\left(2 \mathrm{mg} \mathrm{mL}^{-1}\right)$ dropwise at $98^{\circ} \mathrm{C}$ for a certain time $(2-6 \mathrm{~h}$, the obtained products named RGO (h, h) and RGO (x, h)). Preparation of the TIMs has been described in our previous reports. ${ }^{7,14}$ In the first step, the RGO sample was dispersed in water (lysozyme was added and the $\mathrm{pH}$ value of the solution was adjusted to 10), ${ }^{19}$ and treated with ultrasonication for $10 \mathrm{~min}$. Then, the well-dispersed RGO sample was poured into ER under modest stirring for $10 \mathrm{~min}$. After stirring, the composite was cured at $110{ }^{\circ} \mathrm{C}$ for $2 \mathrm{~h}$. The mass fractions of the RGO filler in the TIMs were $5 \mathrm{wt} \%, 10 \mathrm{wt} \%, 15 \mathrm{wt} \%$ and $20 \mathrm{wt} \%$.

\section{Characterization}

Morphological images were recorded by a scanning electron microscope (SEM, FEI Sirion 200 working at $5 \mathrm{kV}$ ). Raman spectra were obtained by the LabRam-1B Raman microspectrometer at $532 \mathrm{~nm}$. X-ray photoelectron spectroscopy (XPS) profiles were recorded on an RBD upgraded PHI-5000C ESCA system. Infrared spectroscopy (IR) curves were measured on an IR Prestige-21 system. Laser flash analysis and differential scanning calorimetry (Diamond DSC, PerkinElmer) were used to obtain the thermal performance of the composites. The 
mechanical properties of these composites were recorded by a Triton DMTA (Triton Instruments, UK).

\section{Conclusions}

RGO specimens with different ratios of various surface functional groups are prepared and adopted to modify the ER in TIMs, and the resulting thermal and mechanical properties are detected. Among all the surface groups of the RGO samples, the carboxyl group displays the highest ability to act as a bridge to enhance the phonon transport at the interface between the graphene basal plane and ER. After optimizing the morphology, reduction degree and functional group type of the adopted RGO filler, the thermal conductivity of the prepared TIMs reaches $5.9 \mathrm{~W} \mathrm{~m}^{-1} \mathrm{~K}^{-1}$. Moreover, the thermal stability and mechanical performance of the resulting TIMs are also investigated. A high ratio of carboxyl groups is not only beneficial for the high thermal conductivity of the TIMs, but also in favor of a high mechanical performance because of its bonding ability with hydroxyl (and epoxy) groups of the ER.

\section{Conflicts of interest}

There are no conflicts to declare.

\section{Acknowledgements}

This work is supported by National Natural Science Foundation of China (No. 51506012, 61401297, 51574044, and 61701333), Jiangsu Science Foundation Fund (No. BK20150266, BK20140283), Foundation of Key Laboratory in Science and Technology Development Project of Suzhou (No. SZS201609).

\section{Notes and references}

1 H. Yang, C. Shan, F. Li, Q. Zhang, D. Han and L. Niu, J. Mater. Chem., 2009, 19, 8856-8860.

2 C. X. Liu and G. X. Hu, Appl. Therm. Eng., 2015, 90, 193-198. 3 Y. X. Fu, Z. X. He, D. C. Mo and S. S. Lu, Appl. Therm. Eng., 2014, 66, 493-498.

4 L. M. Veca, M. J. Meziani, W. Wang, X. Wang, F. Lu, P. Zhang, Y. Lin, R. Fee, J. W. Connell and Y. P. Sun, Adv. Mater., 2009, 21, 2088-2092.

5 H. S. Sung, H. P. Kwang and H. K. Bo, Adv. Mater., 2013, 25, 732-737.

6 M. F. Khan and A. A. Balandin, Nano Lett., 2012, 12, 861-867.

7 B. Tang, G. X. Hu, H. Y. Gao and L. Y. Hai, Int. J. Heat Mass Transfer, 2015, 85, 420-429.

8 B. Tang, Z. W. Wang, W. Q. Huang, S. Li, T. T. Ma, H. G. Yu and X. F. Li, Nanoscale Res. Lett., 2017, 12, 527-533.

9 B. Tang, H. Q. Chen, Y. F. He, Z. W. Wang, J. Zhang and J. P. Wang, Compos. Sci. Technol., 2017, 150, 54-64.

10 B. Tang and G. X. Hu, Chem. Vap. Deposition, 2014, 20, 14-22. 11 F. Zhao, W. X. Kong, Z. G. Hu, J. D. Liu, Y. F. Zhao and B. Zhang, RSC Adv., 2016, 6, 79028-79036.
12 M. J. Lv, T. Mei, C. A. Zhang and X. B. Wang, RSC Adv., 2014, 4, 9261-9270.

13 M. Fu, Q. Z. Jiao and Y. Zhao, RSC Adv., 2014, 4, 2324223250.

14 Y. F. Sun, B. Tang, W. Q. Huang, S. L. Wang, Z. W. Wang, X. B. Wang, Y. J. Zhu and C. B. Tao, Appl. Therm. Eng., 2016, 103, 892-900.

15 S. Bai and X. P. Shen, RSC Adv., 2012, 2, 64-98.

16 W. S. Hummers and R. E. Offeman, J. Am. Chem. Soc., 1958, 80, 1339.

17 G. X. Zhang, Y. Q. Xu, L. Wang, Y. Kuang and X. M. Sun, Sci. China Mater., 2015, 58, 534-543.

18 C. Xu, R. S. Yuan and X. Wang, New Carbon Mater., 2014, 29, 61-66.

19 B. Tang, G. X. Hu, H. Y. Gao and Z. X. Shi, J. Power Sources, 2013, 234, 60-68.

20 B. Tang, S. L. Wang, J. Zhang, Z. W. Wang, Y. F. He and W. Q. Huang, Int. Mater. Rev., 2017, DOI: 10.1080/ 09506608.2017.1344377.

21 B. Tang, G. J. Ji, Z. W. Wang, H. Q. Chen, X. F. Li, H. G. Yu, S. Li and H. Liu, RSC Adv., 2017, 7, 45280-45286.

22 B. Tang, G. X. Hu and H. Y. Gao, Appl. Spectrosc. Rev., 2010, 45, 369-407.

23 P. K. Sahoo, B. Panigrahy and D. Bahadur, RSC Adv., 2014, 4, 48563-48571.

24 A. Heydari and H. Sheibani, RSC Adv., 2016, 6, 9760-9771. 25 A. Heydari and H. Sheibani, RSC Adv., 2015, 5, 82438-82449. 26 A. Heydari, M. Iranmanesh, F. Doostan and H. Sheibani, Pharm. Chem. J., 2015, 49, 605-612.

27 M. F. Khan and A. A. Balandin, Nano Lett., 2012, 12, 861-867.

28 Z. Fan, F. Gong, S. T. Nguyen and H. M. Duong, Carbon, 2015, 81, 396-404.

29 S. T. Nguyen, H. T. Nguyen, A. Rinaldi, N. P. V. Nguyen, Z. Fan and H. M. Duong, Colloids Surf., A, 2012, 414, 352358.

30 H. M. Duong, D. V. Papavassiliou, K. J. Mullen and S. Maruyama, Nanotechnology, 2008, 19, 065702-065706.

31 M. Mehrali, S. T. Latibari, M. Mehrali, T. M. Mahlia, H. S. Metselaar, M. S. Naghavi, E. Sadeghinezhad and A. R. Akhiani, Appl. Therm. Eng., 2013, 61, 633-640.

32 R. Z. Zhang and W. Chen, J. Mater. Chem. A, 2013, 1, 1145711464.

33 W. L. Xiong, Y. Chen, M. Hao, L. Zhang, T. Mei, J. Y. Wang, J. H. Li and X. B. Wang, Appl. Therm. Eng., 2015, 91, 630-637.

34 S. K. Sahoo, S. Mohanty and S. K. Nayaka, RSC Adv., 2015, 5, 13674-13691.

35 H. Zhang, X. J. Lv, Y. M. Li, Y. Wang and J. H. Li, ACS Nano, 2010, 4, 380-386.

36 X. Wang, W. Y. Xing and P. Zhang, Compos. Sci. Technol., 2012, 72, 737-743.

37 D. G. Cahill, K. Goodson and A. Majumdar, J. Heat Transfer, 2002, 124, 223-241.

38 S. Ghosh, D. L. Nika, E. P. Pokatilov and A. A. Balandin, New J. Phys., 2009, 11, 095012-095020. 